Math. Ann. 285, 700 (1989)

\title{
Erratum
}

\section{On set theoretic complete intersections in $\mathbb{P}^{3}$}

\section{David B. Jaffe}

Department of Mathematics and Statistics, University of Nebraska, Lincoln, NE 68588-0323, USA

Math. Ann. 285, 165 (1989)

In this article the text that should be on p. 174 has mistakenly been printed on p. 175 and vice versa. 